Musées, Patrimoine et Culture scientifiques et techniques

179 | 2018

septembre-octobre 2018

\title{
Décalages, parodies et machines à rire au musée D'Ethnographie de Neuchâtel
}

\section{Grégoire Mayor}

\section{OpenEdition \\ Journals}

Édition électronique

URL : http://journals.openedition.org/ocim/2772

DOI : $10.4000 /$ ocim. 2772

ISSN : 2108-646X

Éditeur

OCIM

Édition imprimée

Date de publication : 1 septembre 2018

Pagination : 28-33

ISSN : 0994-1908

Référence électronique

Grégoire Mayor, « Décalages, parodies et machines à rire au musée D'Ethnographie de Neuchâtel », La Lettre de l'OCIM [En ligne], 179 | 2018, mis en ligne le 01 septembre 2019, consulté le 07 septembre 2019. URL : http://journals.openedition.org/ocim/2772 ; DOI : 10.4000/ocim.2772

Ce document a été généré automatiquement le 7 septembre 2019

Tous droits réservés 


\section{Décalages, parodies et machines à rire au musée D'Ethnographie de Neuchâtel}

\section{Grégoire Mayor}

Dans la salle « Bazars » de l'exposition L'impermanence des choses (2017), la vitrine consacrée aux

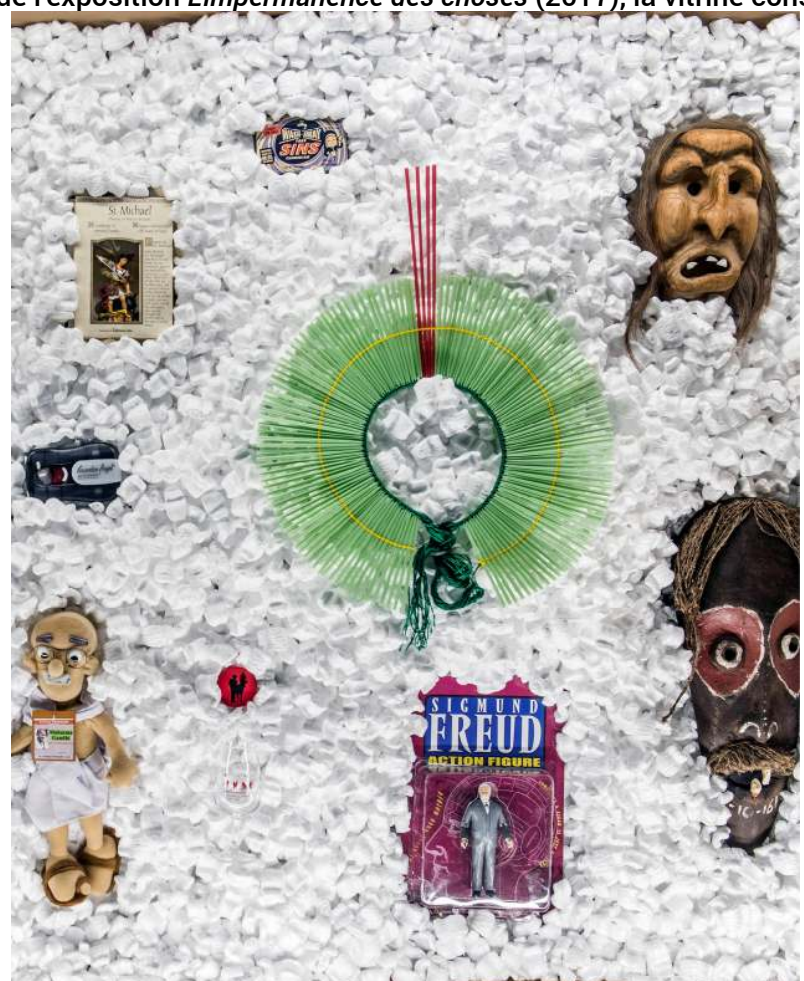

commandes par Internet.e

\section{(c) MEN/Alain Germond}

Peut-on vraiment rire au musée ? La question a longtemps paru incongrue, tant l'institution muséale a été pensée, perçue et vécue comme un temple de la connaissance 
et du sérieux. Si le divertissement y occupe désormais une place plus conséquente, et si la transmission de la connaissance par des dispositifs ludiques est désormais acceptée et valorisée, la présence de l'humour dans les expositions des musées de société n'est pas fréquente. Pourtant, depuis le début des années 1980, les conservateurs du musée d'ethnographie de Neuchâtel (MEN) ne se sont pas privés d'en faire un large usage. Le manifeste écrit par Marc-Olivier Gonseth et Jacques Hainard pour l'exposition La Différence (1995) présente même l'humour comme une des caractéristiques de la distanciation: "exposer, c'est suggérer l'essentiel à travers la distance critique, marquée d'humour, d'ironie et de dérision $»^{1}$.

2 Parodie, ironie, grossissement, caricature, retournement, décalage, glissement sémantique, jeu avec les références, filage ou absurde mise à plat des métaphores... démonter en bon horloger les ressorts et les rouages de l'humour dans les expositions du MEN dépasse le cadre de cet article. Un inventaire détaillé reste à faire, impliquant une réflexion sur les processus créatifs collectifs ainsi que sur la dynamique de l'écart qui est au cœur des mécanismes humoristiques ${ }^{2}$. Sans aller si loin, il faut rappeler ici un paradoxe fondamental : l'humour est très sérieusement exploité au MEN, où il est pensé comme un des moyens à disposition des créateurs d'exposition pour questionner le visiteur, proposer un regard critique sur le monde, voire simplement transmettre des connaissances. Juxtaposer des objets pour créer des significations nouvelles, s'amuser avec les titres, jouer avec les références culturelles, détourner ou parodier des images, des textes, des films ou créer des machines poétiques et réflexives sont quelques-uns des mécanismes humoristiques mobilisés.

\section{Collecter avec humour?}

3 Si l'humour est présent dans les mises en scène du MEN, il l'est aussi dans les logiques de collection. Depuis Objets prétextes, objets manipulés (1984), les objets occidentaux acquis pour les expositions temporaires sont entrés dans les réserves du musée. Signalant des pratiques sociales, économiques et culturelles, ils peuvent prêter à sourire en raison des imaginaires étonnants qu'ils révèlent. Souvent de facture industrielle, leur conservation par un musée d'ethnographie peut paraître incongrue. Dans la salle "Bazars » de L'impermanence des choses (2017), la nouvelle exposition de référence, une série de vitrines présente un résumé des modalités d'acquisition des collections par le musée depuis le début du XXe siècle. La dernière vitrine montre un ensemble d'objets qui ont pour point commun d'avoir été acquis sur l'Internet. Parmi ceux-ci, une action figure représentant Sigmund Freud a été achetée pour un des étals du grand marché de la mémoire de l'exposition Remise en boites (2006), qui portait sur les obsessions commémoratives contemporaines. L'objet lui-même est insolite et drolatique : pour quel consommateur ce petit père articulé de l'inconscient a-t-il en effet été imaginé ? Des collections constituées en dehors des expositions peuvent également témoigner d'un regard amusé sur le fonctionnement de la société. Comme par exemple un ensemble de gadgets promotionnels envoyés aux médecins par des entreprises pharmaceutiques: le petit phoque en peluche ou l'os en gomme frappés au nom des produits que celles-ci cherchent à vendre apparaissent comme bien dérisoires en regard des enjeux financiers considérables de l'économie de la santé. Et c'est justement cette tension, cet écart entre des objets de peu ou de rien et les raisons pour lesquelles ils sont conçus et distribués qui 
crée un effet comique. En plus du fait qu'il est certainement assez unique que de telles productions entrent dans un musée.

L'espace « Chez Happy Daze Lifestyle, c'est l'avenir qui est obsolète » dans l'exposition Remise en boîte (2006).

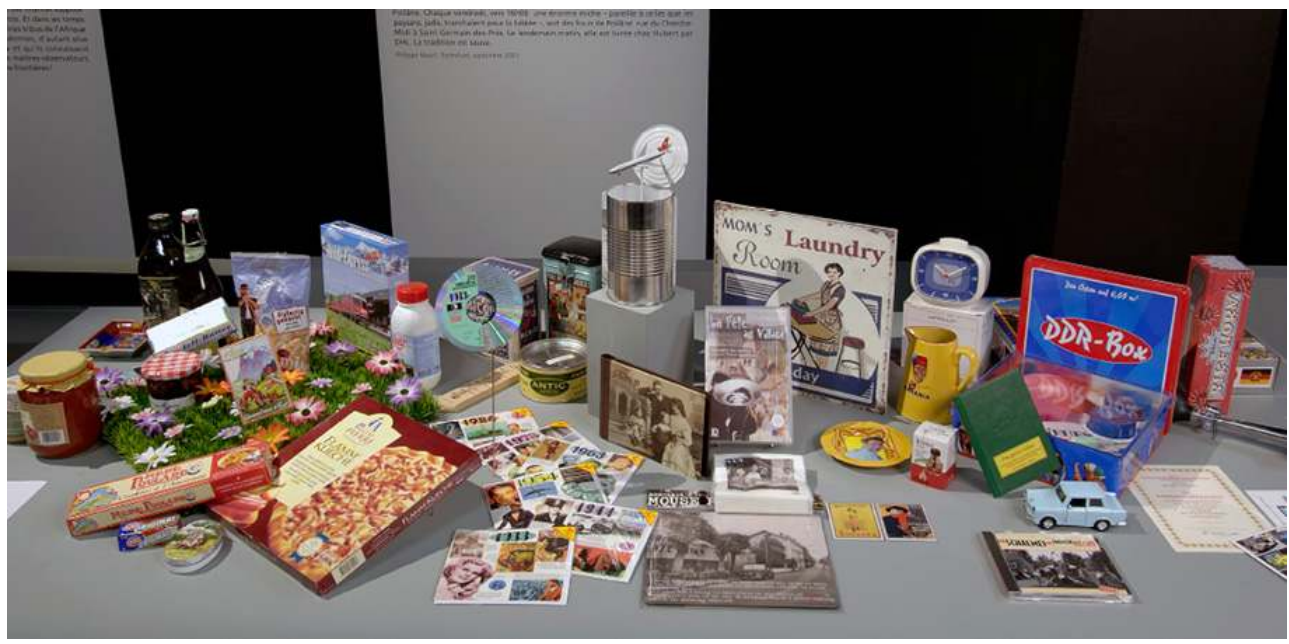

(ㄷ) MEN/Alain Germond

\section{Titrer et décadrer}

Très discutés lors des séances de conception, les titres des expositions et sections qui les composent sont des moyens de signaler des prises de position critiques sur les problématiques abordées. Clins d'œil aux visiteurs, ils peuvent jouer avec les références culturelles, comme La marque jeune (2008), inspiré de la bande dessinée de Blake et Mortimer ou Retour d'Angola (2007), qui rappelle le film Retour d'Afrique d'Alain Tanner (1973). De manière plus décalée, Bruits (2010) invitait à une réflexion sur la patrimonialisation des phénomènes sonores et Hors-Champs à la place de l'image en anthropologie et en muséographie! Après des années de travaux et de déménagements des collections, intituler la nouvelle exposition permanente L'impermanence des choses (2017) peut également apparaître comme une pirouette verbale. Et pourtant ne désigne-telle pas aussi l'étrange folie du projet muséal, cette lutte constante contre les ravages du temps ? Au-delà du titre général, les zones des expositions apparaissent comme de fertiles terrains de jeux sémantiques. Par exemple, les différentes salles de Bruits étaient intitulées les souvenirs de la houle, le bruit des autres, le doux murmure de la théorie, les sirènes de l'urgence, l'écho des réserves et le glouglou des pipe-lines... Les associations inattendues entre les mots, les images et les choses créent des petits courts circuits mentaux qui peuvent libérer le rire. Et cette tension permet certainement de voir le monde différemment, rappelant le projet relativiste de l'anthropologie. En forçant le trait, ils peuvent souligner l'absurdité de certains discours et pratiques ; un flyer disposé sur une des tables du grand marché de la mémoire de Remise en boîtes (2006) donnait ainsi la clé d'interprétation paradoxale de cette collection d'objets glorifiant le passé : «Chez Happy Daze Lifestyle, c'est l'avenir qui est obsolète ». 


\section{Rencontres étonnantes et présences incongrues}

5 Depuis près d'une quarantaine d'années, le MEN propose des mises en situations et en relations inattendues d'objets, de textes et d'images pour que les visiteurs mettent en question leurs certitudes, qu'ils réagissent et s'interrogent (Hainard, 1985). Cette démarche peut déstabiliser ou choquer, mais également faire rire. Dans la salle « Ambassades » de L'impermanence des choses (2017), un couvercle de sarcophage offert à la Suisse par le khédive d'Égypte à la fin du XIXe siècle voisine avec des poupées représentant les nationalités de l'Union soviétique données au musée en pleine guerre froide par l'ambassade d'URSS ainsi qu'avec un nain de jardin bardé de clous transmis au directeur du MEN, Marc-Olivier Gonseth, par sa majesté Helvetus IV, roi des Suisses, lors de l'inauguration d'Helvetia Park en $2009^{3}$. Les objets réunis ici révèlent l'importance de la diplomatie culturelle dans l'histoire du musée, puisque la plupart sont des présents diplomatiques. Pour autant, le grand salon de l'ambassade a été construit en carton et les fenêtres teintées de rose offrent une vision déformée du monde extérieur. Filant la métaphore à partir du lieu mis en scène, des dispositifs techniques inspirés des films d'espionnage sont planqués un peu partout. Donnant sérieusement accès aux archives, ils éclairent avec humour le contexte historique de chaque donation. Enfin, dans le fond de cette salle, un camp de réfugiés a été installé, rappelant que des objets sont arrivés dans des circonstances plus dramatiques liées à des situations de guerre ou de conflits.

6 L'inclusion d'intrus dans une collection ou une scénographie permet souvent de questionner à la fois la thématique de l'exposition et les modalités de la recherche. Par exemple, dans la section de l'exposition Hors-champs (2012) consacrée à l'utilisation des images en anthropologie pour illustrer le geste technique, le " headbanging " - cette rotation de la tête chère aux amateurs de hard-rock qui leur permet de faire tourner leur chevelure en rythme - a été présenté comme un candidat atypique mais plausible à l'inscription au patrimoine culturel immatériel. La dynamique humoristique de l'écart est renforcée ici par l'utilisation d'une formalisation graphique liée à la recherche ethnographique : l'autodérision ou la parodie de la technologie culturelle en anthropologie n'est pas loin. Mais en même temps cette présentation interroge sérieusement sur l'inclusion potentielle d'une telle pratique culturelle comme patrimoine immatériel de l'humanité et évoque le rôle de la description ethnographique comme instance de légitimation dans ce type de processus.

\section{Parodie et autodérision}

7 La parodie fait partie des dispositifs fréquemment utilisés au MEN. L'outil est particulièrement puissant puisqu'il permet d'indiquer des codes culturels et de signaler des conventions esthétiques et narratives dans les œuvres parodiées. Clin d'œil aux visiteurs, elle suppose au moins que ceux-ci puissent identifier les produits culturels en question. La prise de distance, qui porte fréquemment au MEN sur l'anthropologie et la muséographie a ainsi parfois été considérée comme élitiste, hermétique ou marquant un relativisme épistémologique trop forcé : qui croire si même les musées se moquent d'euxmêmes ? Et comment se positionner face à l'avalanche de références mises en scènes et en abîmes ? Le travail sans cesse remis à l'ouvrage de l'équipe de conception et de réalisation est bien de rendre l'image muséographique la plus polysémique possible, de proposer aux 
visiteurs une expérience d'interprétation. Du simple détournement d'affiches publicitaires au "hmokumentary ", les formes de la parodie sont variées. Elles sont le plus souvent réalisées par l'équipe de conception et réalisation (scénographes, graphiste et photographe), mais elles peuvent être également choisies parmi des œuvres artistiques déjà existantes. Dans la plupart des cas, les éléments parodiques sont mobilisés dans le scénario comme contre-points ou comme détournements de représentations sociales et culturelles. Dans Secrets (2015), exposition qui se déployait dans toute la ville de Neuchâtel à la manière d'une chasse au trésor, un court-métrage était ainsi présenté dans une salle cachée du musée d'Art et d'Histoire. Réalisé à l'instigation de la professeure Ellen Hertz et de l'ethnologue et écrivain Nicolas Yazgi, qui a également cosigné le scénario, il poussait la logique patrimoniale à bout en imaginant l'inscription du secret bancaire sur la liste des éléments suisses du patrimoine culturel immatériel. Il s'inscrivait dans une série de réalisations sur des petits métiers traditionnel que j'ai initiée en 2004 ( Le facteur de langue de bois, Le distillateur d'esprit de clocher, L'affineur de film suisse...) et s'inspirait des films de promotion du patrimoine culturel immatériel de l'Unesco.

Le spectateur découvrait les petits métiers menacés par la disparition possible du secret bancaire : l'invisible passeur de valises ; le mutique éleveur de carpes ; la discrète prêteuse de noms et le dynamique entrepreneur de bas de laine helvétique promouvant l'opacité dans un monde de transparence... Outre la parodie de l'esthétique et de la rhétorique urgentiste caractéristiques de nombreux films ethnographiques, l'humour naissait du décalage assumé entre les enjeux de l'économie mondiale et la préservation des petits métiers traditionnels dans un monde rural idéalisé.

Bruits (2010), publicités mettant en avant le patrimoine culturel pour promouvoir une région.

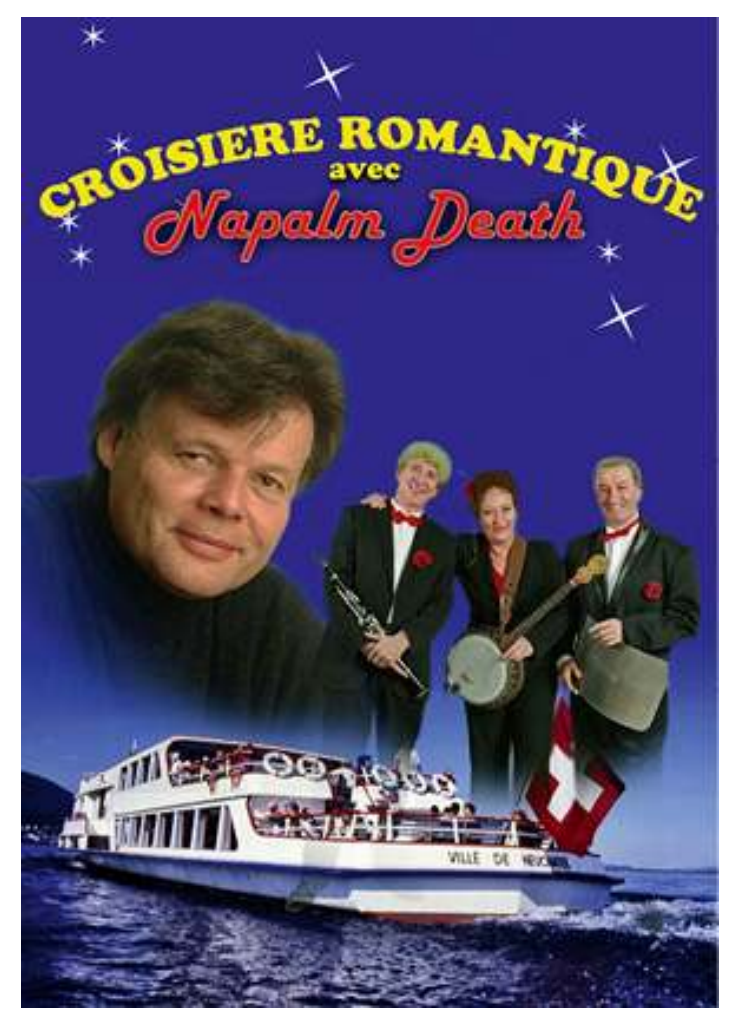




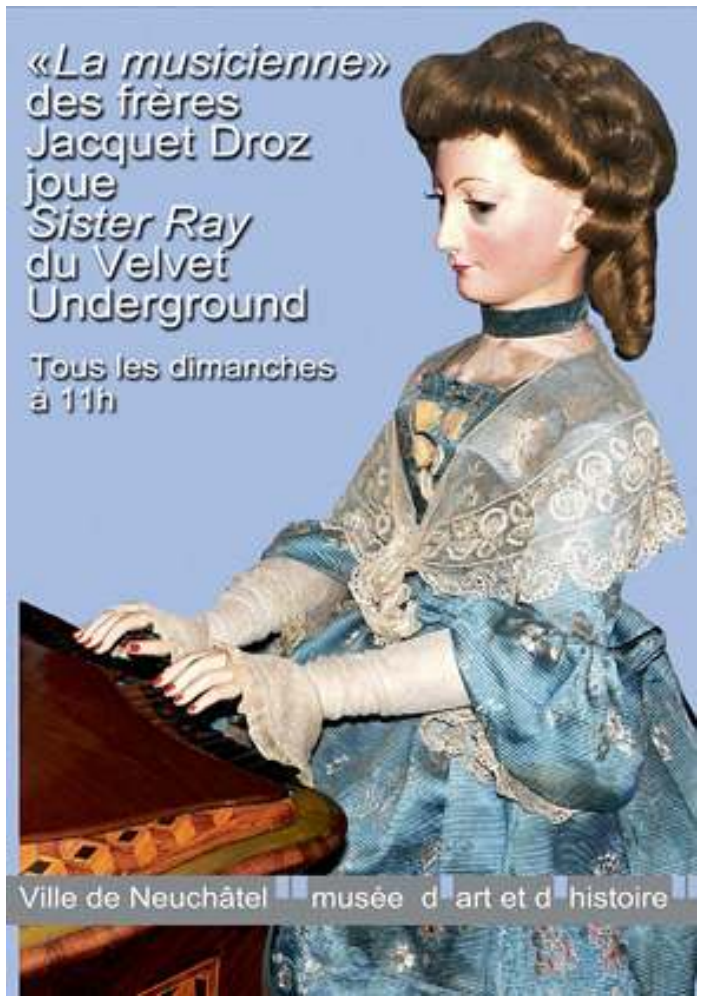

(c) MEN/Alain Germond

Helvetia Park (2009) avait certainement dans sa logique d'ensemble une connotation parodique. Pour traiter des thèmes liés à la culture en Suisse, l'équipe du MEN avait construit en effet un décor de grande fête foraine. Munis d'Heidi, monnaie frappée pour l'occasion, les visiteurs pouvaient flâner du manège des traditions populaires nommé ironiquement Eternal Tour, au train fantôme Teufelsbrüke (le pont du diable) qui rappelait les scandales culturels ayant émaillé l'histoire suisse. Le jeu de massacre Battleground évoquait quant à lui la place et le développement de la critique satirique en Suisse, dans la presse comme dans les émissions radiophoniques. En abattant toutes les têtes, les visiteurs révélaient une belle phrase de Georges Balandier, marquant l'ambivalence et les limites de l'usage de l'humour : "l'imagerie satirique introduit selon sa manière à la connaissance du grand jeu de l'ordre et du désordre, de la conformité et de la contestation. Comme le cérémonial bouffon, elle recourt au renversement des situations, à l'irrévérence et aux licences de toutes sortes. Elle pratique l'offensive en utilisant les forces du comique et du ridicule. Avec la même ambiguité, car elle libère une critique que le rire désamorce " (Balandier,1992). Enfin le champ de foire était dominé par la cabane de la voyante Madame Helvetia, crachant sans cesse des prédictions culturelles aléatoires selon une machine adaptée de l'artiste berlinois Henning Wagenbreth. Dans un monde où les experts en marketing culturel produisent des masses de pronostics de toutes sortes, la comparaison avec la diseuse de bonne aventure ne s'imposait-elle pas? 
«Transmettre : la fondue comme patrimoine culturel immatériel.... référence et dérision en question ». Hors-champs (2012).

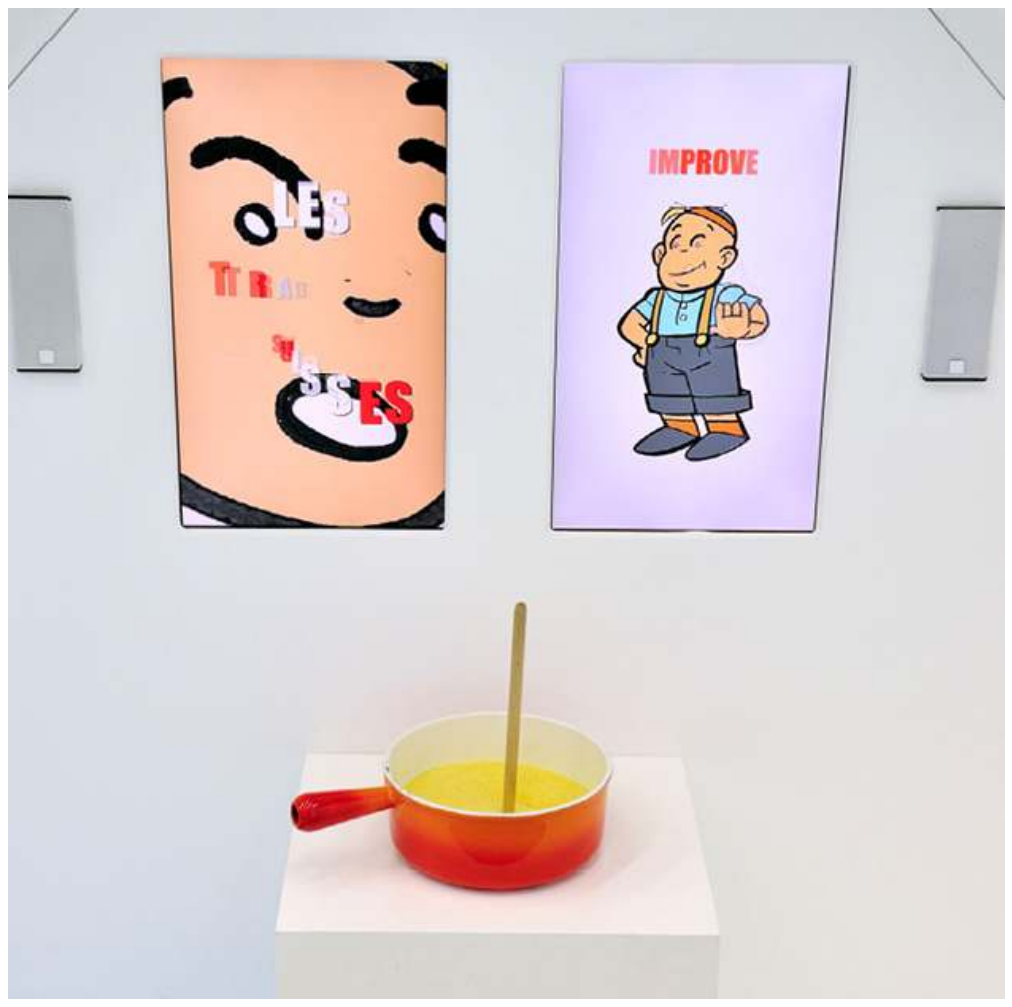

(c) MEN/Alain Germond

\section{Des machines à rire?}

Le détournement de dispositifs techniques a occupé une place de choix dans les expositions des dernières années. Et comme les jeux simples de télescopages d'objets, d'images et de textes, les technologies futures offrent d'infinies possibilités créatrices pour autant qu'on ne les prenne pas trop au sérieux. Dans Hors-champs, un jeu vidéo permettait ainsi au visiteur de s'exercer à brasser la fondue, tout en étant constamment tancé par des experts et théoriciens. Une parodie claire des modules interactifs présents dans les musées des techniques, réalisée ici autour d'un geste très simple puisque il suffisait d'être le plus constant possible pour éviter que la masse de fromage n'attache au caquelon. Pour autant, cette manière ludique de célébrer l'entrée de la fondue comme pratique sociale dans la liste du patrimoine culturel immatériel suisse rappelait que dans la logique d'inscription au PCI, la confection du plat importait moins que les discours avisés qui l'accompagnaient. La machine allait ainsi paradoxalement au-delà de la parodie en remplissant parfaitement sa fonction pédagogique! Toujours dans Hors-champs, un dispositif informatique poussait à l'extrême les logiques d'évaluation dont la société contemporaine raffole et qui se déploient à foison dans les interfaces techniques. Dans une zone de l'exposition consacrée aux usages des images dans les processus classificatoires, le visiteur était poussé à donner son avis sur des représentations de l'altérité anciennes et contemporaines. Ses choix lui donnaient accès à des publicités pour des produits calqués sur ses goûts, cours de danse orientale ou festivals Touaregs dans le désert, avant qu'il ne se retrouve piraté par un hacker lointain, qui lui envoyait une lettre 
de demandes de fonds rédigée en fonction de sa sélection. Ultime pirouette, il se retrouvait lui-même classé par l'institution comme un type de visiteur (" Ours ", " Anguille » et « Rossignol »), à la manière des études des publics des musées. Dans Bruits, deux machines absurdes résumaient deux fonctions fondamentales des musées : une machine à distiller créait de l'esprit à partir de la matérialité, des théories à partir d'objets ; et le "Trad'Icer » congelait les traditions culturelles pour les transformer en boule à neige, condensation dans une installation métaphorique de la crainte de figer des pratiques sociales, du rêve pour certains de les transformer en objets touristiques et du fait que les expositions ne sont en définitive que des constitutions de petits mondes sous cloche!

"Trad'icer », la machine instrallée dans l'exposition Bruits (2010) pour congeler les traditions culturelles.

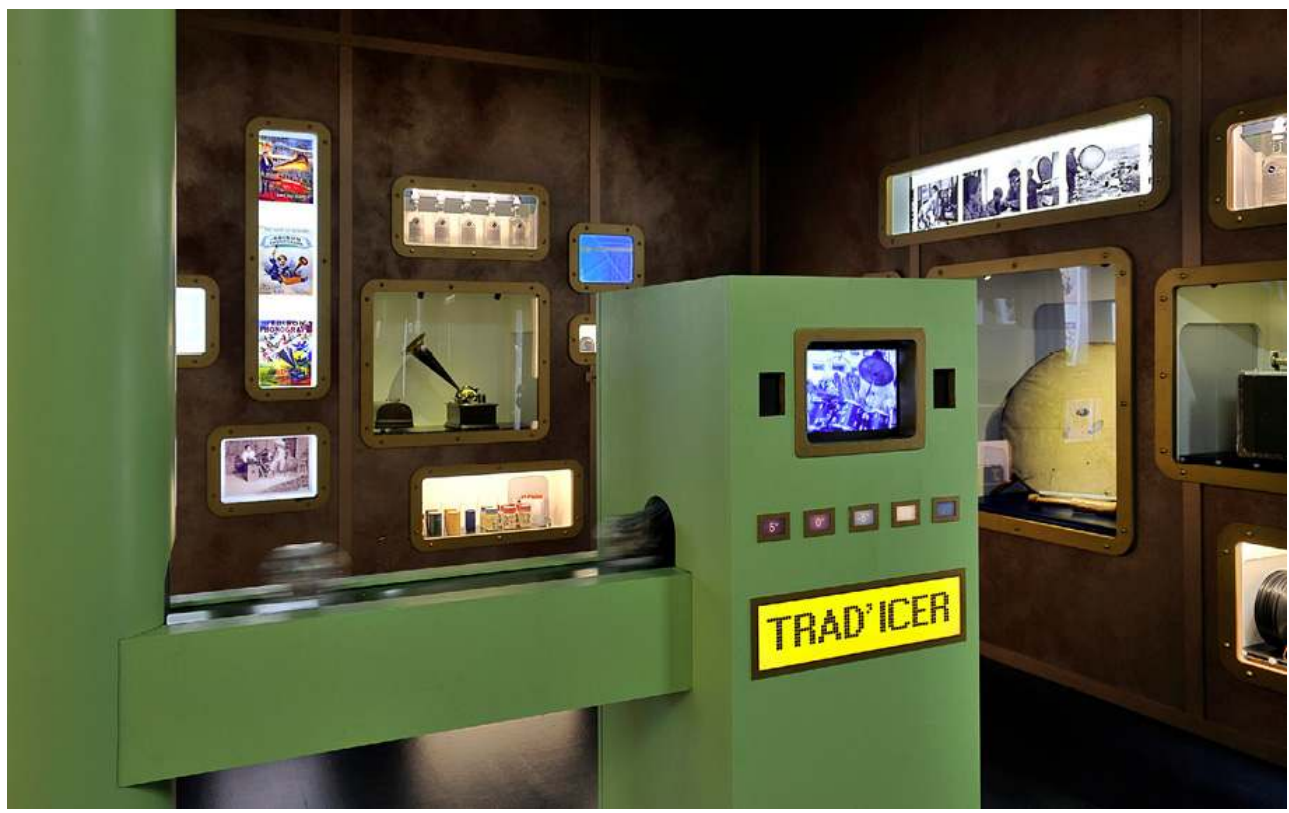

(C) MEN/Alain Germond

11 Comme esquissé dans cet article, l'humour a bien évidement sa place dans la création de ces petits mondes. À l'instar d'autres moyens de communication, il permet de toucher ou déstabiliser les visiteurs, de transmettre des informations tout en gardant une certaine distance par rapport aux sujets d'études des musées de société. Politesse du désespoir selon Chris Marker, l'humour est aussi l'affirmation d'une position de pouvoir. Mais n'estce pas le rôle des concepteurs d'exposition de prendre position ? De ne pas créer dans leur installation de simples reflets de la société mais bien de sans cesse la questionner ? Par ailleurs, faut-il rappeler cette évidence, la vie en société n'est heureusement de loin pas toujours sérieuse et l'humour, la satire, le détournement, la caricature font partie de nombreux comportements sociaux, voire même d'importants rituels comme le carnaval. Certes, la connaissance des codes est indispensable pour mesurer l'écart qui crée la surprise, pour saisir l'ironie ou l'autodérision. Mais une pratique réflexive de la muséographie se doit de jouer avec les références, pour sans cesse déconstruire les manières convenues de penser et d'exposer. Réfléchir avec un amusement sérieux à ses propres comportements comme aux comportements des autres, reste un des moyens les plus efficaces de comprendre le monde. 
Balandier, G. Le pouvoir sur scènes. Paris : Balland, 1992.

Gonseth, M.-O. Un atelier expographique, in Gonseth, M.-O., Hainard, J. et Kaehr, R. (éds.) Cent ans d'ethnographie sur la colline de Saint-Nicolas. Neuchâtel : MEN, 2005, pp. 375-394. Hainard, J. La tentation d'exposer, in Hainard, J. et Kaehr, R. (éds.) Temps perdu, temps retrouvé : voir les choses du passé au présent. Neuchâtel : MEN, 1985, pp. 153-156.

\section{NOTES}

1. www.men.ch/fr/expositions/principes-dexposition/

2. Pour une stimulante analyse des processus créatifs collectifs à l'œuvre au MEN voir MarcOlivier Gonseth, 2005.

3. Le Roi des Suisses évolue dans l'œuvre photoshopée des deux artistes neuchâtelois Plonk et Replonk, qui ont composé la couverture de ce numéro de La Lettre de l'Ocim.

\section{RÉSUMÉS}

Marque de fabrique depuis plus de trente ans des expositions proposées par le musée d'Ethnographie de Neuchâtel, l'humour est utilisé pour interroger le visiteur, tenter de remettre en question ses certitudes et surtout lui permettre d'exercer son esprit critique. L'un des deux responsables de l'établissement présente ici les outils mis en œuvre tout au long de ce processus créatif.

INDEX

Mots-clés : gregoire.mayor@ne.ch

\section{AUTEUR}

\section{GRÉGOIRE MAYOR}

Conservateur au musée d'Ethnographie

de Neuchâtel 